\title{
Negative Ion Fast-Atom Bombardment Tandem Mass Spectrometry To Determine Sulfate and Linkage Position in Glycosaminoglycan- Derived Disaccharides
}

\author{
Diane J. Lamb, Hui M. Wang, Larry M. Mallis*, and Robert J. Linhardt \\ High Resulution Mass Spectrometry Facility and Division of Medicinal and Natural Products Chemistry, \\ College of Pharmacy, University of Iowa, Iowa City, Iowa, USA
}

\begin{abstract}
Negative ion fast-atom bombardment tandem mass spectrometry has been used in the analysis of monosulfated disaccharides. These commercially obtained disaccharides have been enzymatically prepared from glycosaminoglycans using polysaccharide lyases. Three disaccharides from chondroitin sulfate and dermatan sulfate and two disaccharides from heparan sulfate and chemically derivatized heparin were analyzed. All five disaccharides were isomeric, with differences in sulfate position and linkage position. The full-scan mass spectra are useful in differentiating isomers when the sulfate group resides on different saccharide units. This structural information was obtained from fragment ions produced through cleavage at the glycosidic linkage. The full-scan mass spectra of each monosulfated disaccharide also produced intense molecular anions having long lifetimes. Collisional activation of these resulted in tandem mass spectra rich in significant product ions. Some of these fragment ions were formed through ring cleavage and were useful in the determination of both sulfate and linkage position. (J Am Soc Mass Spectrom 1992, 3, 797-803)
\end{abstract}

G lycosaminnglycans (C.AGs) are sulfated, linear polysaccharides that are found in virtually all living tissues [1]. The GAGs differ in types of saccharide constituents, sulfation, and linkage configuration and position. GAGs can be separated into two major families, those with $(1 \rightarrow 4)$ linkages (i.e., heparin and heparan sulfate) and those with alternating $(1 \rightarrow 3,1 \rightarrow 4)$ linkages (i.e., chondroitin and dermatan sulfates). Theses GAGs are composed of alternating, glycosidically linked uronic acid and hexosamine residues. Although the uronic acid residue is only sulfated at $\mathrm{C}-2$, the hexosamine residue can be sulfated at $\mathrm{C}-2, \mathrm{C}-3, \mathrm{C}-4$, and $\mathrm{C}-6$. Both of these families play an important role in cell-cell interaction [2], as well as in a wide variety of other biological functions [3]. Our laboratury is involved in developing new methods to study the structure of these complex polysaccharides [4-6]. We rely on polysaccharide lyases $[7,8]$ to break down these GAGs into smaller oligosaccharides for structure determination. The aim is to relate features in the fine structure of these GAGs, such as the posi-

\footnotetext{
* Current address: Bristol-Myers Squibb Co., Pharmaceutical Re search Institute, New Brunswick, NJ 08903.

Address reprint requests to Robert J. Linhardt, Division of Medicinal and Natural Products Chemistry College of Phanmacy, University of Iowa, Iowa City, IA 52242.
}

tion of sulfate groups, to specific biological activities [9].

Carbohydrate structure determination has increasingly come to rely on mass spectrometry primarily because of its high sensitivity [10]. Mass spectral approaches for the analysis of nonvolatile, highly sulfated oligosaccharides prepared from GAGs often require prior derivatization $[11,12]$. One method to analyze underivatized sodium salts of sulfated oligosaccharides, prepared using polysaccharide lyases, involves the use of negative ion fast-atom bombardment mass spectrometry (FAB-MS). This results in both molecular and fragment aniuns, from the cleavage of glycosidic linkages, that afford sequence information [13, 14]. The fragmentation observed in the negative ion FAB-MS of underivatized samples, however, does not permit the assignment of sulfate position within a saccharide residue nor does it afford information on the linkage position. These are important structural features, since a complex mixture of GAGs is often found in a single biological sample.

Negative ion FAB tandem mass spectrometry (MS/MS) has recently been applied to oligosaccharide analysis and its fragmentation mechanisms and nomenclature discussed [15, 16]. FAB MS/MS of neutral oligosaccharides has shown utility in determining 
linkage position [17, 18] and studies on acidic monosaccharides have shown FAB MS/MS to be useful for determining sulfate position [19]. In this paper we report the negative ion FAB-MS and FAB MS/MS of a series of commercially obtained, GAG-derived disaccharides demonstrating the utility of this method to obtain ions regarding both linkage and sulfate position.

\section{Experimental}

Materials. $\quad$ UUA2S(1 $\rightarrow$ 4)-D-GlcNAc and $\triangle \mathrm{UA}(1 \rightarrow$ 4)-D-GlcNAc6S (where $\triangle \mathrm{UA}$ is a 4-deoxy- $\alpha$-L-threo-hex4 cno pyranosyluronic acid, GlcNAc is $N$-acetylglucosamine, and $S$ is sulfate) were purchased from Grampian Enzymes (Aberdeen, Scotland). $\triangle \mathrm{UA2S}(1 \rightarrow$ 3)-D-GalNAc, $\triangle U A(1 \rightarrow 3)$-D-GalNAc4S, and $\triangle U A(1$ $\rightarrow 3$ )-D-GalNAc6S (where GalNAc is N-acetylgalactosamine) were purchased from Seikagaku America (Ruckville, MD). These disaccharides could also be prepared by treating GAG or chemically derivatized GAG with the appropriate polysaccharide lyase and purifying the desired disaccharide using high-performance liquid chromatography (HPLC) [8, 20]. Triethanolamine (TEA) and sodium dodecylsulfate (SDS) were from Sigma Chemical Co. (St. Louis, MO). The Bio-Gel P2 desalting column was from BioRad (Richmond, CA). Thioglycerol, deuterium oxide (99.99 atom 96) and 3-(trimethylsilyl)propionic acid-2,2,3,3- ${ }^{2} \mathrm{H}_{4}$ sodium salt $\left(\mathrm{TSP}-{ }^{2} \mathrm{H}_{4}\right)$ were from Aldrich Chemical Co. (Milwaukee, WI). Sodium borate was from Fisher Chemical Co. (Fair Lawn, NJ) and boric acid was from Mallinckrodt (Paris, KY).

Characterization of the Disaccharide Standards by ${ }^{1} \mathrm{H}-\mathrm{NMR}$ Spectroscopy. Each disaccharide was dissolved in ${ }_{2}^{2} \mathrm{H}_{2} \mathrm{O}$ and exchanged three times by freeze drying. The ${ }^{1} \mathrm{H}-\mathrm{NMR}$ spectra of each disaccharide $(1.5 \mathrm{mg} / 0.5 \mathrm{~mL})$ was obtained at $360 \mathrm{MHz}$ using a Bruker WM 360 NMR spectrometer (Spectrospin AG, Switzerland) at $25{ }^{\circ} \mathrm{C}$ with $\mathrm{TSP}_{-}{ }^{2} \mathrm{H}_{4}$ as the internal standard. The spectra were assigned on the basis of reported literature values and were consistent with the structures of each disaccharide $[14,21]$. The purity of each disaccharide was estimated by ${ }^{1} \mathrm{H}-\mathrm{NMR}$ based on the absence of signals corresponding to contaminants.

Characterization of the Disaccharide Standards by Capillary Zone Electrophoresis (CZE). CZE analysis was performed using a Dionex capillary electrophoresis system (Sunnyvale, CA) equipped with a variable wavelength ultraviolet detector set at $232 \mathrm{~nm}$. The experiments used a fused silica (externally coated except where the tube passed through the detector) capillary tube ( $75 \mu \mathrm{m}$ i.d., $375 \mu \mathrm{m}$ o.d., $70 \mathrm{~cm}$ long) from Dionex. The capillary tube was washed extensively and activated with $0.1 \mathrm{M}$ phosphoric acid, $0.5 \mathrm{M}$ sodium hydroxide, and deionized water then filled with the operating buffer. The $\Delta \mathrm{UA} 2 \mathrm{~S}(1 \rightarrow 3)$-DGalNAc, $\triangle \mathrm{UA}(1 \rightarrow 3)$-D-GaINAc4S, and $\triangle \mathrm{UA}(1 \rightarrow 3)$ D-GalNAc6S were analyzed using $10 \mathrm{mM}$ sodium borate and $50 \mathrm{mM}$ boric acid at $\mathrm{pH} 8.8$ [22] and $10 \mathrm{kV}$ and the $\Delta U A 2 S(1 \rightarrow 4)$-D-GlcNAC and $\Delta U A(1 \rightarrow 4)$-DGlcNAc6S were analyzed using $50 \mathrm{mM}$ SUS, $10 \mathrm{mM}$ sodium borate at $\mathrm{pH} 8.5$ and $20 \mathrm{kV}$ [21]. The samples were injected by gravity injection ( $1 \mathrm{~nL}$ containing 1 $\mathrm{ng}$ ) and electrophoresis was performed at $25^{\circ} \mathrm{C}$ using the above operating buffers.

Mass Spectrometry. All mass spectra were acquired using a VG ZAB-HF mass spectrometer (VG Analytical Ltd., Manchester, UK) equipped with an Ion Tech saddle-field FAB gun and a standard VG source. The gun was operated at $8 \mathrm{keV}$ with a $2-\mathrm{mA}$ current.

The samples were desalted at least twice using a Bio-Gel P2 column $(35 \mathrm{~cm} \times 2.5 \mathrm{~cm})$ eluted with deionized water. The samples (approximately $1 \mu \mathrm{L}$ of a $20 \mathrm{mg} / \mathrm{mL}$ solution) were dissolved in $2 \mu \mathrm{L}$ TEA (or thioglycerol) and added to a standard VG stainless steel probe tip.

For FAB-MS and FAB MS/MS experiments, ions were produced by bombarding the sample with $8 \mathrm{keV}$ xenon atoms. Precursor ions were selected using the magnetic sector at a mass resolution of 500 and were collisionally activated by introducing helium into the collision cell to reduce the beam of precursor ions by approximately 50\%. Mass-analyzed ion kinetic energy (MIKE) spectra were obtained by scanning the electrostatic analyzer and averaging 8 scans with the use of the multichannel analysis software on the VG 11-250J data system. The detector dynode was set at $7 \mathrm{kV}$ and positioned for optimal sensitivity across the entire energy range [23].

\section{Results and Discussion}

The two families of GAGs found in tissues that are most often associated with important biolugical activities are the heparin/heparan sulfate and the chondroitir/dermatan sulfates [1]. This study used commercially available disaccharide standards prepared from GAGs and chemically modified GAGs that had been depolymerized using various polysaccharide lyases [7], fractionated, and purified by $\operatorname{HPLC}[8,20]$. Each disaccharide was dissolved in deionized, distilled water to give a concentrated $(20 \mathrm{mg} / \mathrm{mL}$ ) stock solution. A portion of this stock solution was used directly for CZE and FAB-MS analysis, while a second portion was exchanged with ${ }^{2} \mathrm{H}_{2}$ for ${ }^{1} \mathrm{H}-\mathrm{NMR}$ spectroscopy. NMR analysis at $360 \mathrm{MHz}$ resulted in spectra consistent with structure for each of the five monosulfated disaccharides $[14,21]$. The purity of each was $>90 \%$ by NMR. CZE analysis gave electropherograms that showed a single symmetrical peak for each of the disaccharides indicating a purity of $>95 \%[21,22]$. The purity of these disaccharides had also been previ- 
ously established by strong anion exchange HPLC [24] and reversed phase ion pairing HPLC analysis [25].

Studies by our group have demonstrated that it is possible to analyze the underivatized sodium salts of GAG-derived disaccharides by FAB-MS [13, 14] and to obtain both molecular anion and fragment ions resulting in both structure and sequence information. The analysis of thoroughly desalted samples using triethanolamine as the liquid FAB matrix gave more intense spectra and more fragmentation than other commonly used matrices, such as thioglycerol [13]. All five of the GAG-derived, monosulfated disaccharides gave negative ion $F A B$ spectra with very intense molecular anion peaks at $m / z 458$ and $480\left(\left[\mathrm{M}-\mathrm{H}^{-}\right.\right.$ and $[\mathrm{M}+\mathrm{Na}-2 \mathrm{H}]^{-}$) with a low intensity molecular anion at $m / z 502\left([\mathrm{M}+2 \mathrm{Na}-3 \mathrm{H}]^{-}\right)$(where $\mathrm{M}$ is the fully protonated molecule).

Two difficulties remain for this analytical approach: one is the presence of matrix ions that can confuse the interpretation of spectral data; the second is the low amount of fragmentation that occurs, making full structural assignment difficult. Ions arising from the triethanolamine matrix can be subtracted out, howr ever, giving spectra that are easier to interpret (Figure 1). The ring-cleavage fragments, crucial for the assignment of structure, are generally not observed. Our previous studies [13, 14] identified several GAG-derived oligosaccharides that were difficult to distinguish when analyzed by F $\Lambda B-M S$. These problems are particularly apparent when two isomers are analyzed. Isomeric disaccharides can differ in both sulfate and linkage position yet show very similar $\mathrm{FAB}$ mass spectra. It is possible to distinguish between monosulfated disaccharides only when the sulfates are on different saccharide units (i.e., $2 S$ from $4 S$ and 6S, Figure 1). No significant differences are observed in the spectra of monosulfated disaccharides isomers having sulfates on the same saccharide unit (i.e., $4 S$ from $6 S$ ). For example, while the spectra of $\triangle \mathrm{UA2S}(1 \rightarrow 3)$-D-GalNAc and $\triangle \mathrm{UA2S}(1 \rightarrow 4)$-D-GlcNAC contained an ion at $m / z 277$ corresponding to $[\mathrm{M}+\mathrm{Na}-\mathrm{H}-\mathrm{GalNAc}]$ or $[\mathrm{M}+$ $\mathrm{Na}-\mathrm{H}-$ GlcNAcl $^{-}, \Delta \mathrm{UA}(1 \rightarrow 3)$-D-GalNAc4S, $\triangle \mathrm{UA}(1 \rightarrow 3)$-D-GalNAc6S, and $\triangle \mathrm{UA}(1 \rightarrow 4)$-DGlcNAc6s contained ions at $m / z 300$ corresponding to [M- $\Delta U A]$ (Figure 1). In addition, differences in linkage positions (i.e., $1 \rightarrow \mathbf{3}$ and $1 \rightarrow 4$ ) can not be distinguished from the full-scan FAB mass spectra of monosulfated disaccharides (Figure 1).

Because all the monosulfated disaccharides produce very intense $\left[\mathrm{M}-\mathrm{H}^{-}\right.$and $[\mathrm{M}+\mathrm{Na}-2 \mathrm{H}]^{-}$ions having long lifetimes, their metastable [26] and collision-induced dissociation (CID) MIKE spectra were recorded. Collision gas was not required to obtain tandem mass spectra rich in structurally significant product ions; however, collisional activation restulted in some structurally significant ions that were not observed in the metastable MIKE spectra.

The CID-MIKE spectra of monosaccharidic sulfated glucosamines have been reported to contain fragment
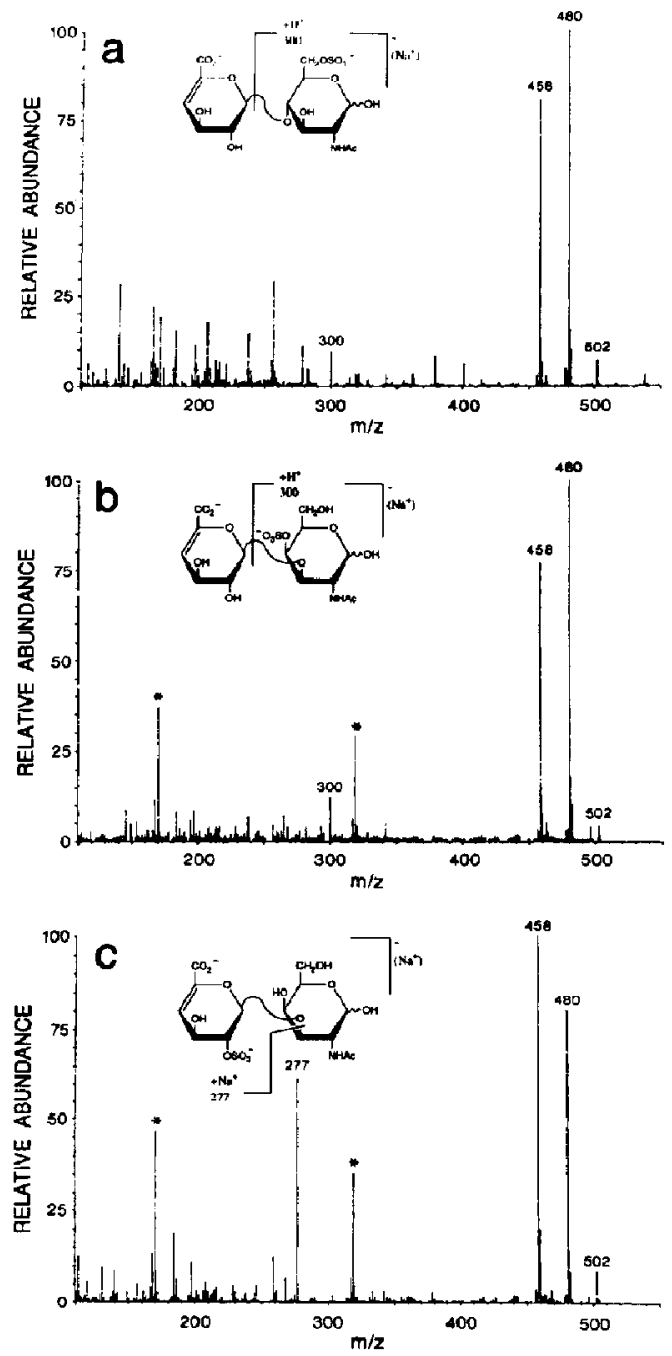

Figure 1. Full-scan negative-ion FAB spectra of (a) $\triangle U A(1 \rightarrow 4)$ D-GleNACGS, (b), $\triangle U A(1 \rightarrow 3)$-D-GaINAc4S, and (c) $\triangle U A 2 S(1 \rightarrow$ 3)-D-GalNAc. The structures are presented in their polyanionic form $(M-2 H)$. Matrix ions have been subtracted in (a) whereas the asterisks in (b) and (c) mark matrix ions and sodium ion clusters not removed by matrix subtraction.

ions from which the sulfate position can be determined [19]. Thus, we obtained the CID-MIKE spectra of [M $\mathrm{H}]^{-}$ions $(m / z$ 458) of monosulfated disaccharides in an effort to distinguish sulfate position (Figure 2). Whenever possible, we use the nomenclature recently proposed by Domon and Costello [16] to describe the fragment ions drawn in each figure.

The ${ }^{0.2} \mathrm{X}_{2}$ ion observed at $m / z \quad 342$ and the [HSO $\left.]_{4}\right]^{-}$ ion at $m / z 97$ are common to the [M-H] ${ }^{-}$CID-MIKE spectra for each isomeric disaccharide (Figure 2). Characteristic ions for both $\triangle$ UA2S $(1 \rightarrow 3)$-D-GalNAc and $\triangle \mathrm{UA2S}(1 \rightarrow 4)$-D-GlcNAc (Figure $2 \mathrm{a}$ and d) are the $\mathrm{B}_{1}$, $m / z 237$ and 157 ions corresponding to a cleavage of 

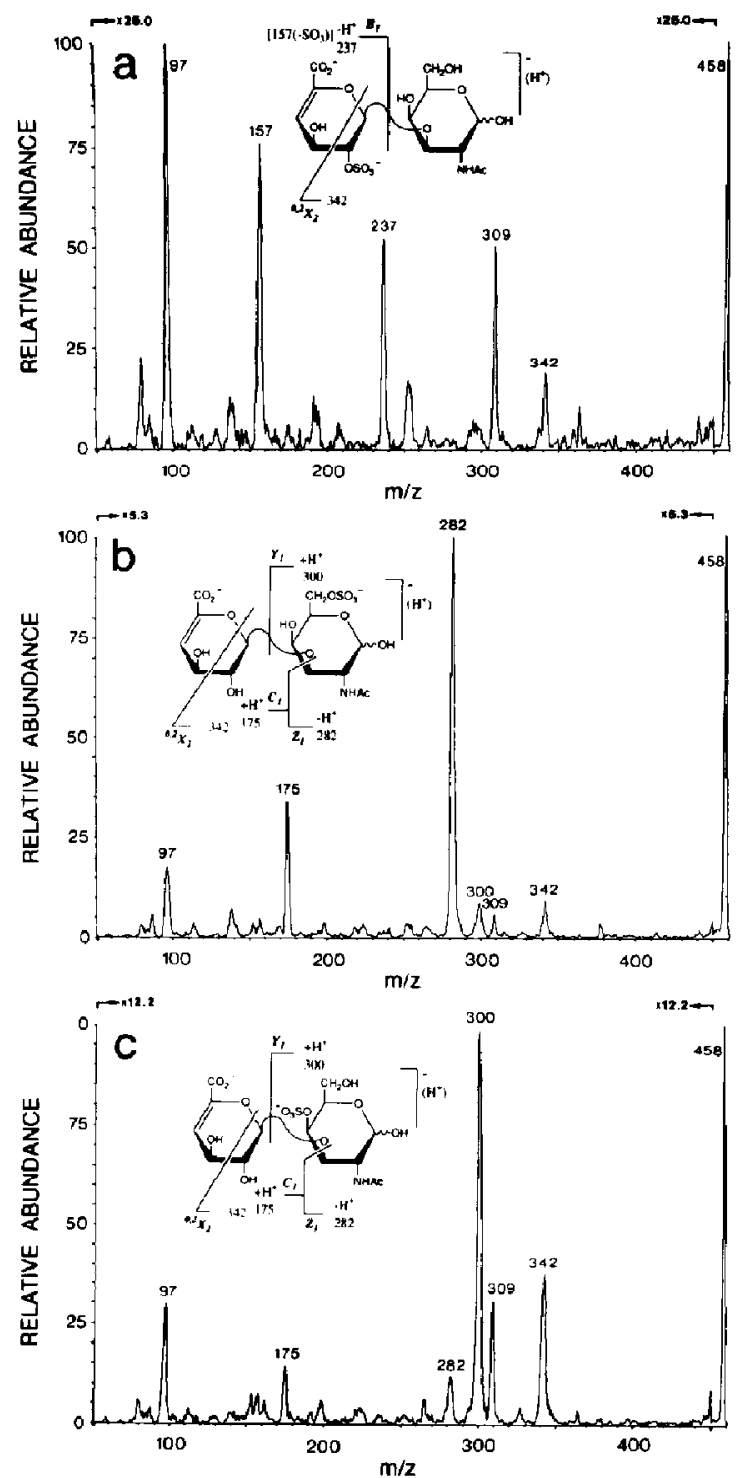
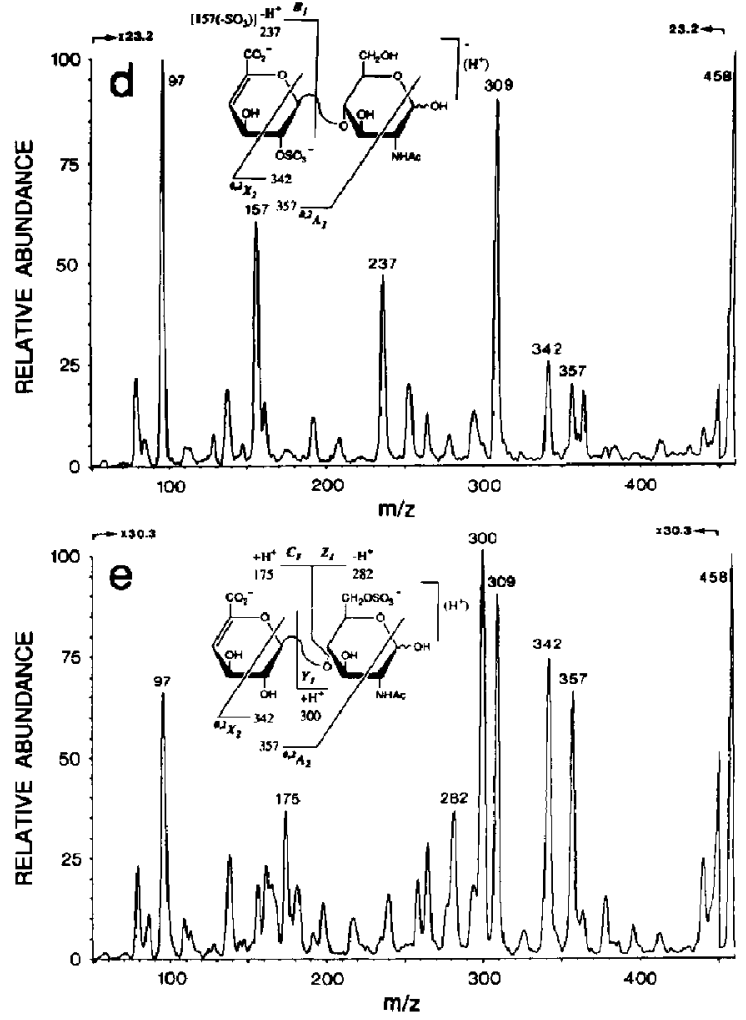

Figure 2. The negative-ion CID-MIKE spectra of $m / z 458,\left([\mathrm{M}-\mathrm{H})^{-}\right)$(a) $\triangle \mathrm{UA2S}(1 \rightarrow 3)$-DGalNAc, (b) $\triangle U A(1 \rightarrow$ 3)-D-GalNAc6S, (c) $\triangle$ UA(1 $\rightarrow$ 3)-D-GalNAc4S, (d) $\triangle U A 2 S(1 \rightarrow 4)$-D-GlcNAc, and $(e) \Delta \mathrm{UA}(1 \rightarrow 4)$-D-GilcNAc6S.

the glycosidic linkage. Although fragment ions corresponding to cleavage through the glycosidic linkage are also observed in the CID-MIKE spectra of $\triangle$ UAC 1 $\rightarrow$ 3)-D-GalNAc4S, $\Delta \mathrm{UA}(1 \rightarrow 3)$-D-GalNAc6S, and $\triangle \mathrm{UA}(1 \rightarrow 4)$-D-GlcNAc6S disaccharides at $m / z 175$, 282 , and 300 from $[M-H]$, it is possible to ditterentiate the $\triangle \mathrm{UA}(1 \rightarrow 3)$-D-GalNAc4S and $\Delta \mathrm{UA}(1 \rightarrow 3)$-DGalNAc6S isomers by the dramatic differences in ion intensities. For example, in the spectrum of $\triangle \mathrm{UA}(1 \rightarrow$ 3)-D-GalNAc4S (Figure 2c), glycosidic cleavage gives the most abundant $Y_{1}$ product ion at $m / z 300$ ([M $\left.\Delta \mathrm{UA}^{-}\right)$. For $\Delta \mathrm{UA}(1 \rightarrow 3)$-D-GalNAc6S, the fragmenta- tion without retention of the glycosidic oxygen gives $Z$ ion formation corresponding to $\mathrm{m} / \mathrm{z} 282$ ([M $\Delta \mathrm{UAJ}^{-}$) as the most abundant process (Figure $2 \mathrm{~b}$ ). This is not surprising because the mechanism for the formation of the $Z$ ion requires an oxygen ion at the 4 position to undergo epoxide formation prior to expulsion of the nonreducing end sugar and $\mathrm{H}$ transfer [27]. It should be noted that $\triangle \mathrm{UA}(1 \rightarrow 3)$-D-GalNAc6S, $\triangle \mathrm{UA}(1 \rightarrow 3)$-D-GalNAc4S, and $\triangle \mathrm{UA}(1 \rightarrow 4)$-DGlcNAc6S fragment to give $C$ and $Z$ ions at $m / z 175$ ([M - GalNAc6S or $4 S]^{-}$or [M - GlcNAc6S $\left.]^{-}\right)$and $m / z \quad 282\left([\mathrm{M}-\Delta \mathrm{UA}]^{-}\right)$that arise from a sul- 
fated saccharide present at the disaccharide's reducingend sugar (Figure $2 b, c$, and e). The absence of the same $C$ and $Z$ ions in the spectra of $\triangle \mathrm{UA2S}(1 \rightarrow 3)$-DGalNAC and $\triangle \mathrm{UA2S}(1 \rightarrow 4)$-D-GlcNAc (Figure $2 \mathrm{a}$ and d) offers an additional feature to distinguish these monosulfated disaccharides.

A peak at $m / z 309$ that corresponds to the loss of $149 \mu$ was observed in several of the CID-MIKE mass spectra (Figure 2 a-e). In thioglycerol the $\mathrm{m} / \mathrm{z} 309$ peak is not observed, suggesting that this peak was due to the TEA $\left(M_{\mathrm{r}}=149\right)$ liquid matrix.

The $[\mathrm{M}+\mathrm{Na}-2 \mathrm{H}]^{-}$ion at $m / z 480$ gives product
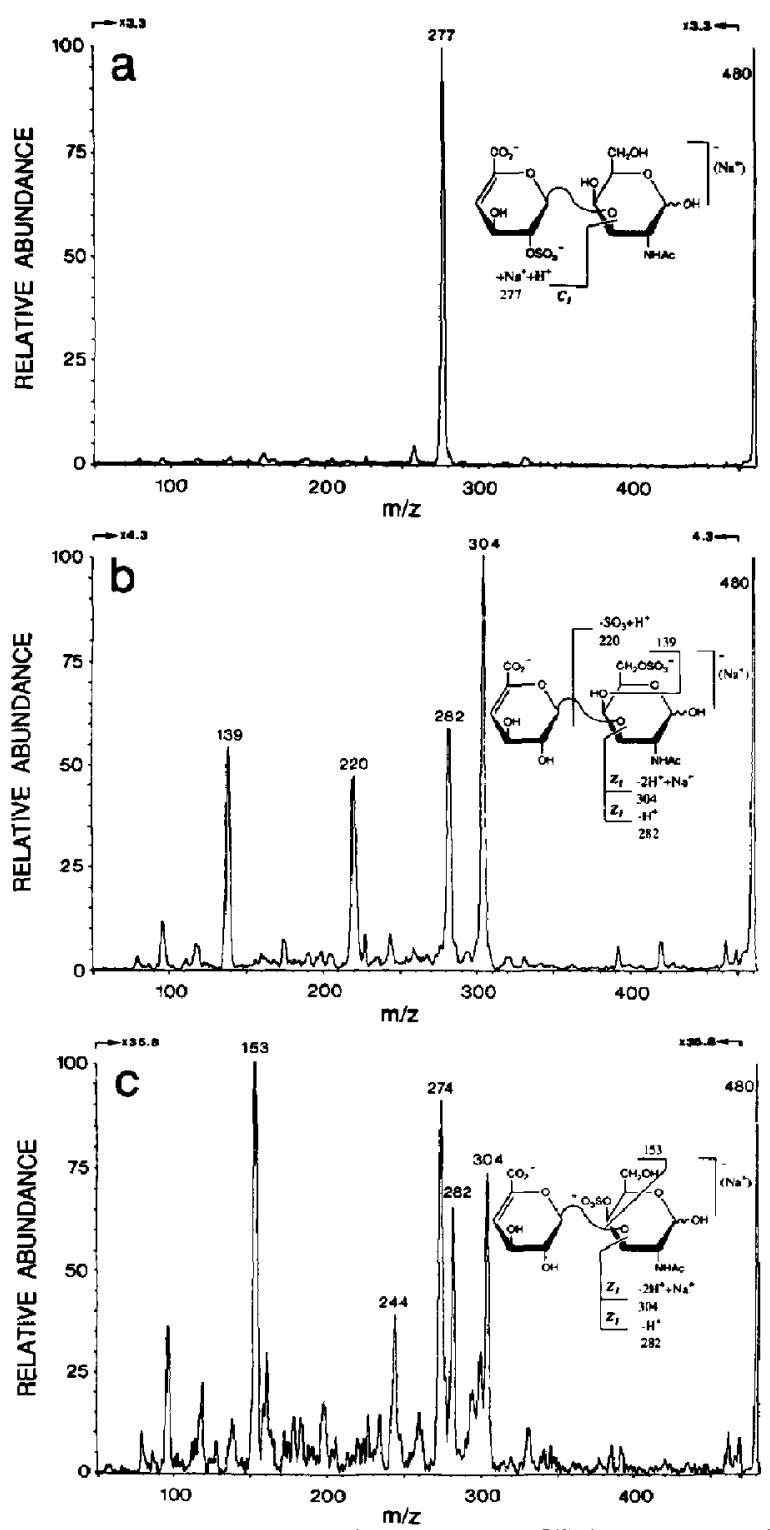

Figure 3. The negative-ion CID-MIKE spectra of $m / z 480$, $\left([\mathrm{M}+\mathrm{Na}-2 \mathrm{H}]^{-}\right)$(a) $\Delta \mathrm{UA2S}(1 \rightarrow 3)-\mathrm{D}-$ GalNAc, (b) $\triangle \mathrm{UA}(1 \rightarrow$ 3)-D-GalNAc6S, (c) $\triangle \mathrm{UA}(1 \rightarrow$ 3)-D-GalNAc4S, (d) $\triangle \mathrm{UA2S}(1 \rightarrow$ 1)-D-GlcNAc, and $(e) \triangle U A(1 \rightarrow 4)$-D-GlcNAc6S. ions in the FAB CID-MIKE spectra that can also be used to deduce sulfate position (Figure 3 ). Positive ion FAB analysis of carbohydrates has shown that the alkali metal cationized species undergo fragmentations that are more structurally informative than the fragmentation of the protonated molecular ion [28]. Negative ions of carbohydrates containing sodium cations have not been as thoroughly studied as nonsodiated ions, so that a well-defined nomenclature is not available. A nomenclature similar to that proposed by Domon and Costello [16] is used for these fragment ions arising from the $m / z 480$ molecular anion. Each
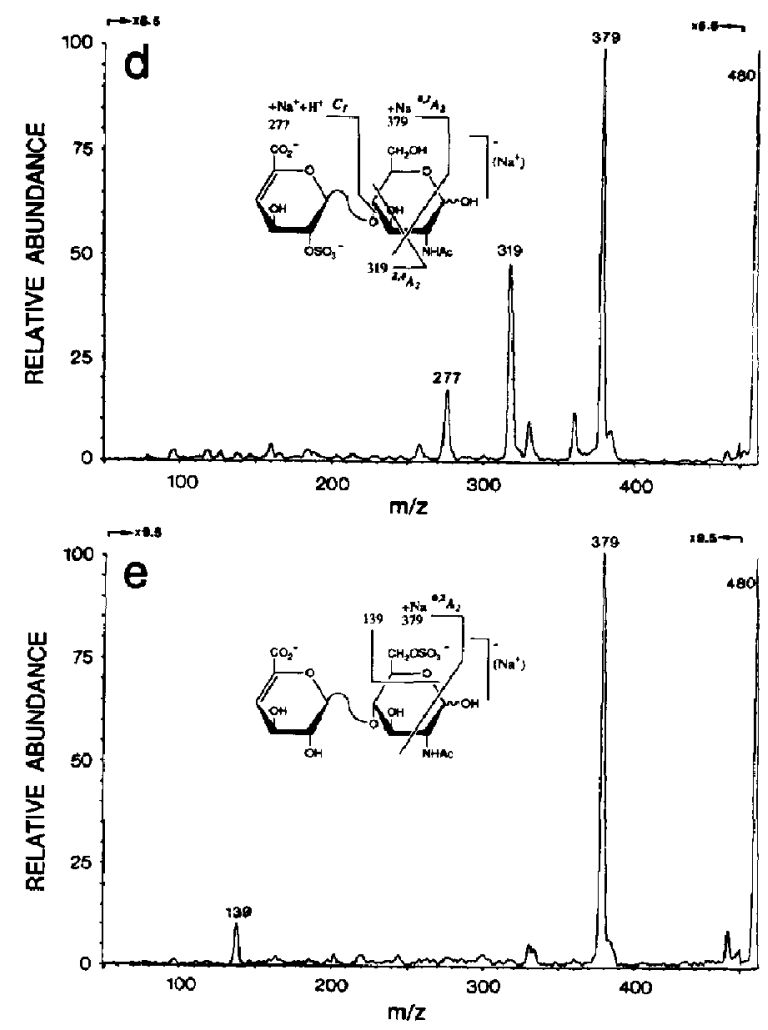
CID-MIKE spectrum arising from the $[\mathrm{M}+\mathrm{Na}-2 \mathrm{H}]^{-}$ precursor ion at $m / z 480$ is unique. For example, ring cleavage results in fragment ions that can be used to distinguish the position of sulfate groups. In these spectra, the presence of an ion at $\mathrm{m} / \mathrm{z} 153$ is only observed for $\triangle \mathrm{UA}(1 \rightarrow 3)$-D-GalNAc4S (Figure 3c) while an ion at $m / z 139$ is characteristic of $\triangle \mathrm{UA}(1 \rightarrow$ 3)-D-GalNAc6S and $\triangle U A(1 \rightarrow 4)$-D-GlcNAc6S (Figure $3 \mathrm{~b}$ and e). It is interesting to note that these two peaks, which contain the sulfate group and require ring cleavage, do not appear in the corresponding metastable spectra [26]. The FAB CID-MIKE spectra of the $\triangle \mathrm{UA2S}(1 \rightarrow 3)$-D-GalNAC and $\triangle \mathrm{UA}(1 \rightarrow 4)$-DGlcNAc6S are simple, containing few fragment ions (Figure $3 a$ and e). The $\triangle$ UA2S $(1 \rightarrow 3)$-D-GalNAc gave $\mathrm{C}_{1}$-type fragmentation with proton and sodium addition, resulting in an abundant product ion at $m / z 277$ $\left(\left[\mathrm{M}-\mathrm{GalNAc}^{-}\right.\right.$) (Figure 3a). The remaining product ions obtained from the $[\mathrm{M}+\mathrm{Na}-2 \mathrm{H}]^{-}$ion of other isomers are quite complex. Many contain sodium and appear to arise from subsequent fragmentation of $\mathrm{Z}$ ions. The ion at $m / z 244$ can be assigned to ring cleavage of the $\mathrm{Z}_{1}$ ion and loss of $\mathrm{HOCH}_{2} \mathrm{CH}-\mathrm{O}$ and the ion at $m / z 274$ to loss of $\mathrm{CH}_{2} \mathrm{O}$ by cleavage of the $C-5-C-6$ bond of the hexosamine residue in the $Z_{1}$ ion (Figure 3c).

When all the GAGs present within a tissue are depolymerized using a mixture of polysaccharide lyases, disaccharides containing both $(1 \rightarrow 3$ and $1 \rightarrow 4)$ glycosidic linkages are obtained. When these are isomeric they are often difficult to separate and analyze [21, 22]. Therefore a tandem mass spectral method capable of distinguishing between such isomeric disaccharides would be very useful. Tandem CID positivemode FAB-MS has been useful in distinguishing linkage position in neutral oligosaccharides (see ref; 28 and references therein).

It is not surprising, therefore, that similar results for sulfated sugars in the negative-ion mode are observed. The ${ }^{0,2} \mathrm{~A}_{2}$ fragment of deprotonated ions is characteristic of the $(1 \rightarrow 4)$ linkage [18]. This is true of the CID-MIKE spectra of either the $\left[\mathrm{M}-\mathrm{H}^{-}\right.$or the $[\mathrm{M}+$ $\mathrm{Na}-2 \mathrm{H}]^{-}$ion of monosulfated disaccharides (Figures 2 and 3). The ${ }^{0,2} A_{2}$ product ion indicative of a $(1 \rightarrow 4)$ linkage is at $m / z 357$ in the [M-H] spectra and at $m / z 379$ in the $[\mathrm{M}+\mathrm{Na}-2 \mathrm{H}]^{-}$spectra (Figures 2 and $3, \mathrm{~d}$ and e). These ions at $m / z 357$ and 379 are not found in the spectra of any of the $(1 \rightarrow 3)$ monosulfated disaccharides.

\section{Conclusions}

In conclusion, FAB CID-MIKE analysis can be used to distinguish both sulfate position and linkage position in GAG-derived monosulfated disaccharides. There are several areas that require future attention. First, the mechanism of sodium interaction with these oligosaccharides as they undergo fragmentation requires additional study. Second, the effect of disulfation on fragmentation also requires further investigation. To answer this question the isomeric disulfated disaccharides of the structure $\triangle \mathrm{UA2S}(1 \rightarrow 4)$-D-GicNAc6S, $\triangle \mathrm{UA2S}(1 \rightarrow 3)$-D-GalNAc6S, and $\triangle \mathrm{UA2S}(1 \rightarrow 3)$-DGalNAc4S are currently under investigation in our laboratory using FAB CID-MIKE scanning. Third, the use of chromatographic [29] and electrophoretic [30] methods combined with tandem FAB-MS, such as that successfully applied to nucleosides and peptides, needs to be explored for acidic carbohydrates. Such approaches are particularly useful when only minor differences are observed in the spectra of isomers. Finally, application of this technique to larger oligosaccharides needs to be examined. We have reported the use of FAB-MS to determine structures as large as a dodecasulfated octasaccharide [14]. The low-intensity molecular anion of this octasarcharide will probably require larger amounts of this highly purified sample for analysis. The application of MS/MS to oligosaccharides, such as this octasaccharide, might be very important in helping to determine the sequence of the GAGs.

\section{Acknowledgments}

We thank Dr. G. Dolnokowski for helpful suggestions during the course of this work. This work was supported in part by the National Institutes of Health (grants GM 38060 and AI 22350). Funds to purchase the mass spectrometer used in these studies and for the support of LMM and DIL were provided by the Office of the University of lowa Vice President for Research.

\section{References}

1. Fransson, L. A. In The Polysaccharides, Vol. 3; Aspinall, G. O. Ed.; Academic Press: New York, 1987; pp. 1-470.

2. Ruoslahti, E. J. Biol. Chem. 1989, 264, 13369-13371.

3. Lane, D. A., Lindahl, U., Eds. Heparin: Chemical and Biological Properties, Clintical Application; Edward Arnold: London, 1989.

4. Rice, K. G.; Rottink, M. D.; Linhardt, R. J. Biochem. J. 1987, $244,515-522$.

5. Loganathan, L;; Wang, H. M.; Mallis, L. M.; Linhardt, R. J. Biochemistry 1990, 29, 4362-4368.

6. Linhardt, R. J.; Rice, K. G.; Kim, Y. S.; Lohse, D. L.; Wang, H. M.; Loganathan, D. Biochem. I. 1988, 254, 781-787.

7. Linhardt, R. J; Cooney, C. L.; Galliher, P. M. Appl. Biochem. Biotech. 1986, 12, 135-177.

8. Rice, K. G.; I.inhardt, R. I. Carhohydr. Res. 1989, 790, 219-233.

9. Atha, D. H.; Lormeau, J. C.; Petitou, M.; Rosenberg, R. D.; Choay, J. Biochemistry 1985, 24, 6723-6729.

10. McCloskey, J. A., Ed. Methods in Enzymology: (Mass Spectrometry), Vol. 193; Academic Press: San Diego, 1990, pp. 1-960.

11. Ballou, C. E.; Dell, A. Carbohydr. Res, 1985, 140, 139-143.

12. Dell, A.; Rogers, M. E.; Thomas-Oates, J. E; Huckerby, T. N.; Sanderson, $1^{3}$. N.; Nieduszynski, I. A. Carbohydr. Res. 1988, 779, 7-19.

13. Mallis, L. M.; Wang, H. M.; Loganathan, D; Linhardt, R. J. Anal. Chem. 1989, 61, 1453-1458.

14. Linhardt, R. J.; Wang, H. M.; Loganathan, D.; Lamb, D. J.; Mallis, L. M. Carbohydr. Res. 1992, 225, 137-145.

15. Dallinga, J. W.; Heerma, W. Biol. Mass Spectrom. 1991, 20, 215-231. 
16. Domon, B.; Costello, C. E. Glycoconjugate J. 1988, 5, 397-409.

17. Laine, R. A.; Pamidimukkala, K. M.; French, A. D.; Hall, R. W.; Abbas, S. A.; Jain, R. K.; Matta, K. L. J. Am. Chem. Soc. 1988, 110, 6931-6939.

18. Garozzo, D.; Giuffrida, M.; Impallomeni, G. Anal. Chem. 1990, 62, 279-286.

19. Dallinga, J. W.; Rinkema, F. D.; Heerma, W. Biomed. Environ. Mass Spectr. 1989, 18, 241-246.

20. Moffat, C. F.; McLean, M. W.; Long, W. F.; Williamson, F. B. Eur. J. Biochem. 1991, 197, 449-460.

21. Ampofo, S. A.; Wang, H. M.; Linhardt, R. J. Anal. Biochem. 1992, 199, 249-255.

22. Al-Hakim, A.; Linhardt, R. J. Anal. Biochem. 1991, 195, 68-73.

23. Savickas, P. J. Rev. Sci. Instrum. 1991, 62, 77-80.
24. Linhardt, R. J.; Al-Hakim, A.; Liu, S. Y.; Kim, Y. S.; Fareed, J. Sem. Thrombosis Hemostas. 1991, 17, 15-22.

25. Linhardt, R. J.; Gu, K. N.; Loganathan, D.; Carter, S. R. Anal. Biochem. 1989, 181, 288-296.

26. Unpublished results.

27. Barber, M.; Bordoli, R. S.; Sedgwick, R. D.; Vickerman, J. C. I. Chem. Soc. Faraday Trans. 1 1982, 78, 1291-1296.

28. Hofmeister, G. E.; Zhou, Z.; Leary, J. A. I. Am. Chem. Soc. 1991, 113, 5964-5970.

29. Jajoo, H. K; Bennett, S. M.; Kornhauser, D. M.; Lietman, P. S.; Cotter, R. J. Proceedings of the 39th ASMS Conference on Mass Spectrometry and Allied Topics; 1991; pp. 1348-1349.

30. 7.11, A.; Zeece, M. G.; Gross, M. L; Cemy, R. L.; Tehrani, J.; Algaier, J. Proceedings of the 39th ASMS Conference on Mass Spectrometry and Allied Topics; 1991; pp. 1374-1375. 